\title{
Estimation of Acceleration Amplitude of Vehicle by Back Propagation Neural Networks
}

\author{
Mohammad Heidari ${ }^{1}$ and Hadi Homaei ${ }^{2}$ \\ ${ }^{1}$ Mechanical Engineering Group, Aligudarz Branch, Islamic Azad University, P.O. Box 159, Aligudarz, Iran \\ ${ }^{2}$ Faculty of Engineering, University of Shahrekord, P.O. Box 115, Shahrekord, Iran
}

Correspondence should be addressed to Mohammad Heidari; moh104337@yahoo.com

Received 5 April 2013; Accepted 19 May 2013

Academic Editor: Emil Manoach

Copyright ( 2013 M. Heidari and H. Homaei. This is an open access article distributed under the Creative Commons Attribution License, which permits unrestricted use, distribution, and reproduction in any medium, provided the original work is properly cited.

\begin{abstract}
This paper investigates the variation of vertical vibrations of vehicles using a neural network (NN). The NN is a back propagation $\mathrm{NN}$, which is employed to predict the amplitude of acceleration for different road conditions such as concrete, waved stone block paved, and country roads. In this paper, four supervised functions, namely, newff, newcf, newelm, and newfftd, have been used for modeling the vehicle vibrations. The networks have four inputs of velocity $(V)$, damping ratio $(\zeta)$, natural frequency of vehicle shock absorber $\left(w_{n}\right)$, and road condition (R.C) as the independent variables and one output of acceleration amplitude (AA). Numerical data, employed for training the networks and capabilities of the models in predicting the vehicle vibrations, have been verified. Some training algorithms are used for creating the network. The results show that the Levenberg-Marquardt training algorithm and newelm function are better than other training algorithms and functions. This method is conceptually straightforward, and it is also applicable to other type vehicles for practical purposes.
\end{abstract}

\section{Introduction}

Recently, improveing comfort and safety conditions for vehicles considering disturbances due to road roughness has been studied by several researchers. To minimise the disturbing effects of vibration, optimum damping factor has been investigated. In the case of definite road profile, that is, for the case of definite vibration with single, two, and three degrees of freedom systems, physical and mathematical models can be established. However, in practice, vehicle vibrations arising from road roughness possess random character. Vibration analysis for such systems can be accomplished by random theory based on statistics. A method which can simulate the set vibrations of vehicle has been developed by Guclu and Gulez [1]. In their investigation, neural network control for a nonlinear full vehicle model was defined by using permanent magnet synchronous motor. Chaos and bifurcation in nonlinear vehicle model have been studied by Li et al. [2], Zhu and Ishitobi [3], and Litak et al. [4]. A solving method of lowfrequency vehicle vibration problems has been presented by Ishihama et al. [5]. Two ideas have been employed. The first was the phase control on vibration transmission in hydraulic engine methods. The other was the vector synthesis approach in treating multiple vibrations input to the vehicle body. A new method for predicting vibration characteristics of a structure that is considered to undergo a design change has been presented [6]. Methodologies for determining the vibration characteristics of the modified structure have also been discussed. A vehicle-subgrade model of vertical coupled system has been presented, and the interactions between the vehicle tuning quality and the subgrade design parameters have been investigated in systematic concept and from the viewpoint of systematic matching [7].

A method for the analysis and simulation of nonstationary random vibrations has been presented by Rouillard and Sek [9]. Their method pays particular attention to the nonstationary nature of vibrations generated by transport vehicles. The limitations of current methods used for analysing and simulating nonstationary random vehicle vibrations were also demonstrated. Yildirim and Uzmay used a radial basis neural network to predict the amplitude of acceleration of vehicle under different road conditions $[10,11]$. 
TABle 1: Parameters depending on road conditions [8].

\begin{tabular}{|c|c|c|c|c|c|c|}
\hline Road & $A_{1}$ & $A_{2}$ & $a_{1}\left(\mathrm{~m}^{-1}\right)$ & $a_{2}\left(\mathrm{~m}^{-1}\right)$ & $b_{2}\left(\mathrm{~m}^{-1}\right)$ & $\sigma_{x 0}(\mathrm{~m})$ \\
\hline \multirow{2}{*}{ As. (R1) } & 0.85 & 0.15 & 0.2 & 0.05 & 0.60 & $0.0080-0.0126$ \\
\hline & 0 & 1 & & 0.22 & 0.44 & 0.12 \\
\hline ESBP (R2) & 1 & 0 & 0.45 & - & - & $0.0135-0.0225$ \\
\hline WSBP (R3) & 0.85 & 0 & 0.45 & - & - & $0.0250-0.0380$ \\
\hline $\mathrm{BP}(\mathrm{R} 4)$ & 0 & 1 & - & 0.32 & 0.64 & 0.017 \\
\hline \multirow{2}{*}{ CR (R5) } & 0 & 1 & - & 0.47 & 0.94 & 0.019 \\
\hline & 0 & 1 & - & 0.11 & 0.146 & $0.067-0.227$ \\
\hline CO (R6) & 1 & 0 & 0.15 & - & - & $0.005-0.0124$ \\
\hline
\end{tabular}

Co.: concrete; As.: asphalt; ESBP: even stone block paved; WSBP: waved stone block paved; BP: boulder paved; CR: country road.

In this study, vertical vehicle vibrations are studied using random theory, and some back propagation artificial neural networks (ANNs) with four functions such as newff, newelm, newcf, and newfftd are also employed to predict amplitudes of accelerations of vehicles for different road conditions.

The organization of the paper is as follows. Section 2 describes the theory of random vibration for vehicles. Overview of neural network is presented in Section 3. More details of modeling of vehicle vibrations using neural networks are given in Section 4. The simulation results obtained form BP are given in Section 5. The paper is concluded with Section 6.

\section{Random Vibration Theory}

Vehicle vibrations due to road roughness have no definite character, and system dynamics depends on the profile of roughness. Therefore, statistical basis random theory is employed in determining roughness character. Assuming such vehicle vibrations to be linear, dynamic model of these systems can be represented as $[12,13]$

$$
\ddot{x}+2 \zeta w_{n} \dot{x}+w_{n} x=-\ddot{x}_{0}(t),
$$

where $x$ is the relative displacement of vehicle body; $x_{0}(t)$ is the amplitude over a specific level of the road roughness on which vehicle's tyre moves at a definite time $t$; $\zeta$ is the damping ratio, and $w_{n}$ also denotes natural frequency of vehicle's shock-absorber system.

By determining amplitudes over a reference plane level on a certain road condition by means of repeated measurements, statistical roughness features are obtained. The road roughness can be determined with enough approximation by some measurements accomplished for different road conditions. In order to describe the influence of the road roughness, the most appropriate statistical parameter is its spectral density, which is a mean square value of road roughness in a definite frequency range. When a vehicle moves at velocity $V$, the road roughness spectral density can be written as follows [12]:

$$
\begin{aligned}
& S_{x_{0}}(w) \\
& \quad= \begin{cases}\frac{2 \sigma_{x_{0}}^{2}}{\pi}\left[\frac{A_{1} \alpha_{1}}{w^{2}+\alpha_{1}^{2}}+\frac{A_{2} \alpha_{2}\left(w^{2}+\alpha_{2}^{2}+\beta_{2}^{2}\right)}{\left(w^{2}-\alpha_{2}^{2}-\beta_{2}^{2}\right)+4 \alpha_{2}^{2} w^{2}}\right] & |w|<w_{1} \\
0 & |w|<w_{1},\end{cases}
\end{aligned}
$$

where $A_{1}+A_{2}=1, \alpha_{1}=a_{1} V, \alpha_{2}=a_{2} V, \beta_{2}=b_{2} V, w_{1}=$ $\Omega V$. As shown in (2), if the spectral density of the road roughness is explained in terms of excitation frequency $w_{1}$, namely, $w$, it is described as $x_{0}^{2} / w=m^{2} s$, or if it is written in terms of the length frequency $\Omega(1 / \mathrm{m})$, it is also described as $x_{0}^{2} / \Omega=m^{3}$. Some parameters depending on road conditions are shown in Table 1 . These given parameters are the results of an experimental investigation [12].

The frequency of vehicle's shock absorber must be chosen between body frequency of $1-1.5 \mathrm{~Hz}$ and axle frequency of $10-15 \mathrm{~Hz}$. Therefore, damping ratio has to be selected so that the frequency of shock absorber is in the range of 4$6 \mathrm{~Hz}$. Consequently, the damping factor for absorber may be taken as a value $<0.5$. In this damping ratio interval $(0.1-$ 0.5 ), vehicle body accelerations decrease for different road conditions [14].

\section{Overview of Neural Networks}

A neural network is a massive parallel system comprised of highly interconnected, interacting processing elements, or nodes. Neural networks process through the interactions of a large number of simple processing elements or nodes, also known as neurons. Knowledge is not stored within individual processing elements, rather represented by the strengths of the connections between elements. Each piece of knowledge is a pattern of activity spread among many processing elements, and each processing element can be involved in the partial representation of many pieces of information. In recent years, neural networks have become a very useful tool in the modeling of complicated systems because they have an excellent ability learn and to generalize (interpolate) the complicated relationships between input and output variables. Also, the ANNs behave as model free 


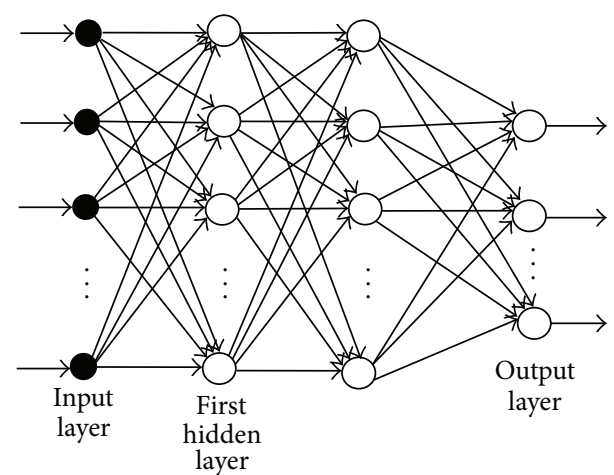

FIGURE 1: Back propagation neural network with two hidden layers.

estimators; that is, they can capture and model complex input-output relations without the help of a mathematical model [15]. In other words, training neural networks, for example, eliminates the need for explicit mathematical modeling or similar system analysis. This property of ANNs is extremely useful in a situation where it is hard to derive a mathematical model. As a result, neural networks can provide an effective solution to solve problems that are intractable or cumbersome with mathematical approaches.

3.1. Back Propagation (BP) Neural Network. The back propagation network (Figure 1) is composed of many interconnected neurons or processing elements (PEs) operating in parallel and are often grouped in different layers.

As shown in Figure 2, each artificial neuron evaluates the inputs and determines the strength of each through its weighing factor. In the artificial neuron, the weighed inputs are summed to determine an activation level. That is,

$$
\operatorname{net}_{j}^{k}=\sum_{i} w_{j i}^{k} o_{i}^{k-1}
$$

where $\operatorname{net}_{j}^{k}$ is the summation of all the inputs of the $j$ th neuron in the $k$ th layer, $w_{j i}^{k}$ is the weight from the $i$ th neuron to the $j$ th neuron, and $o_{i}^{k-1}$ is the output of the $i$ th neuron in the $(k-1)$ th layer.

The output of the neuron is then transmitted along the weighed outgoing connections to serve as an input to subsequent neurons. In the present study, a hyperbolic tangent, log-sigmoid, and linear functions $\left(f\left(\right.\right.$ net $\left.\left._{j}^{k}\right)\right)$ with a bias $b_{j}$ are used as an activation function of hidden and output neurons. Therefore, output of the $j$ th neuron $o_{j}^{k}$ for the $k$ th layer can be expressed as

$$
\begin{gathered}
o_{j}^{k}=f\left(\text { net }_{j}^{k}\right)=\frac{e^{\left(\text {net }_{j}^{k}+b_{j}\right)}-e^{-\left(\text {net }_{j}^{k}+b_{j}\right)}}{e^{\left(\text {net }_{j}^{k}+b_{j}\right)}+e^{-\left(\text {net }_{j}^{k}+b_{j}\right)}} \quad \text { (tansig) }, \\
o_{j}^{k}=f\left(\text { net }_{j}^{k}\right)=\frac{1}{1+e^{- \text {net }_{j}^{k}+b_{j}}} \quad(\text { logsig }), \\
o_{j}^{k}=f\left(\text { net }_{j}^{k}\right)=\text { net }_{j}^{k}+b_{j} \quad \text { (linear) } .
\end{gathered}
$$

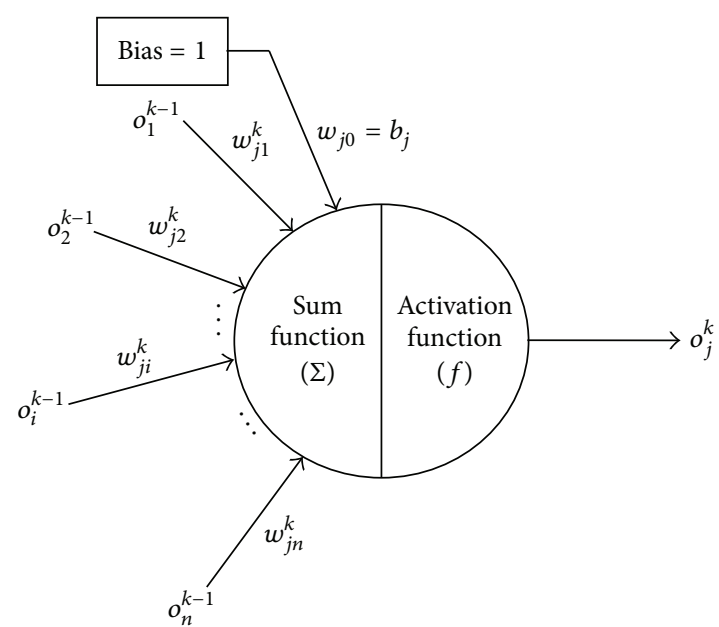

Figure 2: Architecture of an individual PE for BP network.

Before practical application, the network has to be trained. To properly modify the connection weights, an error-correcting technique, often called as back propagation learning algorithm or generalized delta rule [16], is employed. Generally, this technique involves two phases through different layers of the network. The first is the forward phase, which occurs when an input vector is presented and propagated forward through the network to compute an output for each neuron. During the forward phase, synaptic weights are all fixed. The error obtained when a training pair (pattern- " $p$ ") consists of both input and output given to the input layer of the network is expressed by the following equation:

$$
E_{p}=\frac{1}{2} \sum_{j}\left(T_{p j}-O_{p j}\right)^{2},
$$

where $T_{p j}$ is the $j$ th component of the desired output vector, and $O_{p j}$ is the calculated output of $j$ th neuron in the output layer. The overall error of all the patterns in the training set is defined as mean square error (MSE) and is given by

$$
E=\frac{1}{p} \sum_{p=1}^{n} E_{p}
$$

where $n$ is the number of input-output patterns in the training set. The second is the backward phase which is an iterative error reduction performed in the backward direction from the output layer to the input layer. In order to minimize the error, $E$, as rapidly as possible, the gradient descent method adding a momentum term is used. Hence, the new incremental change of weight $\Delta w_{j i}^{k}(m+1)$ can be

$$
\Delta w_{j i}^{k}(m+1)=-\eta \frac{\partial E}{\partial w_{j i}^{k}}+\alpha \Delta w_{j i}^{k}(m),
$$

where $\eta$ is a constant real number between 0.1 and 1 , called learning rate, $\alpha$ is the momentum parameter usually set to a number between 0 and 1 , and $m$ is the index of iteration. 
Therefore, the recursive formula for updating the connection weights becomes

$$
w_{j i}^{k}(m+1)=w_{j i}^{k}(m)+\Delta w_{j i}^{k}(m+1) .
$$

These corrections can be made incrementally (after each pattern presentation) or in batch mode. In the latter case, the weights are updated only after the entire training pattern set has been applied to the network. With this method, the order in which the patterns are presented to the network does not influence the training. This is because of the fact that adaptation is done only at the end of each epoch. And thus, we have chosen this way of updating the connection weights [17].

\section{Modeling of Vehicle Vibrations Using Neural Networks}

Modeling of vehicle vibrations with BP neural network is composed of two stages: training and testing of the networks with numerical data. The training data consisted of velocity $(V)$, damping ratio $(\zeta)$, natural frequency of vehicle shock absorber $\left(w_{n}\right)$, road condition (R.C), and the corresponding acceleration amplitude. A total of 90 data sets were used, of which 80 were selected randomly and used for training purposes whilst the remaining 10 data sets were presented to the trained networks as new application data for verification (testing) purposes. Thus, the networks were evaluated using data that had not been used for training. Before the ANN could be trained and the mapping learnt, it is important to process the numerical data into patterns. Training/testing pattern vectors are formed, each formed with an input condition vector

$$
P_{i}=\left[\begin{array}{c}
\text { velocity }(V) \\
\text { damping ratio }(\zeta) \\
\text { natural frequency }\left(w_{n}\right) \\
\text { road condition }(\text { R.C) }
\end{array}\right]
$$

and the corresponding target vector

$$
T_{i}=[\text { amplitude of acceleration (AA) }] .
$$

Mapping each term to a value between -1 and 1 , we use the following linear mapping formula:

$$
N=\frac{\left(R-R_{\min }\right) *\left(N_{\max }-N_{\min }\right)}{\left(R_{\max }-R_{\min }\right)}+N_{\min },
$$

where $N$ is normalized value of the real variable; $N_{\min }=$ -1 and $N_{\max }=1$ are minimum and maximum values of normalization, respectively; $R$ is real value of the variable; $R_{\min }$ and $R_{\max }$ are minimum and maximum values of the real variable, respectively. These normalized data was used as the inputs and output to train the ANN. Figure 3 shows the general network topology for modeling vehicle vibration.

The names of training algorithms used in this paper are shown in Table 2.

In what follows, the use of four neural networks will be discussed and the results are presented. Then, the best model is picked based on the accuracy of AA in the verification stage.

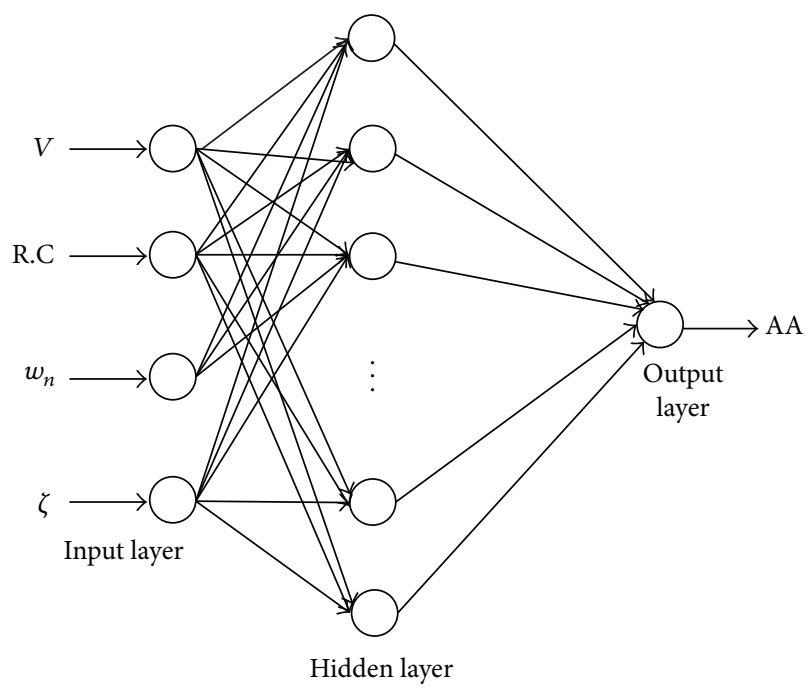

FIgure 3: General ANN topology.

TABLE 2: The variable training methods.

\begin{tabular}{ll}
\hline Acronym & Description \\
\hline LM & Levenberg-Marquardt \\
BFG & BFGS Quasi-Newton \\
RP & Resilient back propagation \\
SCG & Scaled Conjugate Gradient \\
CGB & Conjugate Gradient with Powell/Beale Restarts \\
CGF & Fletcher-Powell Conjugate Gradient \\
CGP & Polak-Ribiére Conjugate Gradient \\
OSS & One Step Secant \\
GDX & Variable Learning Rate back propagation \\
\hline
\end{tabular}

\section{Numerical Results of BP Neural Network Model}

The size of hidden layer(s) is one of the most important considerations when solving actual problems using multilayer feed-forward network. However, it has been shown that BP neural network with one hidden layer can uniformly approximate any continuous function to any desired degree of accuracy given an adequate number of neurons in the hidden layer and the correct interconnection weights [18]. Therefore, one hidden layer was adopted for the BP model. To determine the number of neurons in the hidden layer, a procedure of trial and error approach needs to be done. As such, attempts have been made to study the network performance with a different number of hidden neurons. Hence, a number of candidate networks are constructed, each of trained separately, and the "best" network was selected based on the accuracy of the predictions in the testing phase. It should be noted that if the number of hidden neurons is too large, the ANN might be overtrained giving spurious values in the testing phase. If too few neurons are selected, the function mapping might not be accomplished due to undertraining [19]. Table 3 shows 10 numerical data sets, used 
TABLE 3: Vibration conditions for verification analysis.

\begin{tabular}{lccccc}
\hline $\begin{array}{l}\text { Test } \\
\text { no. }\end{array}$ & $\begin{array}{c}\text { Velocity } \\
(\mathrm{m} / \mathrm{sec})\end{array}$ & $\begin{array}{c}\text { Damping } \\
\text { ratio }(\zeta)\end{array}$ & $\begin{array}{c}\text { Road } \\
\text { condition }\end{array}$ & $\begin{array}{c}\text { Natural } \\
\text { frequency } \\
(\mathrm{Hz})\end{array}$ & $\begin{array}{c}\text { Acceleration } \\
\text { amplitude } \\
(\mathrm{cm})\end{array}$ \\
\hline 1 & 12 & 0.20 & $\mathrm{R} 1$ & 10 & 2.35 \\
2 & 15 & 0.33 & $\mathrm{R} 2$ & 12 & 3.85 \\
3 & 24 & 0.45 & $\mathrm{R} 5$ & 15 & 5.28 \\
4 & 35 & 0.50 & $\mathrm{R} 3$ & 15 & 4.44 \\
5 & 18 & 0.60 & $\mathrm{R} 6$ & 8 & 2.90 \\
6 & 50 & 0.65 & $\mathrm{R} 1$ & 10 & 1.27 \\
7 & 60 & 0.85 & $\mathrm{R} 3$ & 13 & 4.18 \\
8 & 40 & 0.25 & $\mathrm{R} 4$ & 12 & 2.41 \\
9 & 27 & 0.55 & $\mathrm{R} 4$ & 10 & 5.47 \\
10 & 19 & 0.75 & $\mathrm{R} 6$ & 10 & 3.04 \\
\hline
\end{tabular}

TABLE 4: The effect of different number of hidden neurons on the BP network performance.

\begin{tabular}{lcc}
\hline $\begin{array}{l}\text { No. of hidden } \\
\text { neurons }\end{array}$ & $\begin{array}{c}\text { Epoch with LM } \\
\text { method training }\end{array}$ & $\begin{array}{c}\text { Average error in AA (\%) } \\
\text { with newelm function }\end{array}$ \\
\hline 4 & 14390 & 9.80 \\
5 & 5170 & 11.87 \\
$\mathbf{6}$ & $\mathbf{1 7 5 3}$ & 4.95 \\
7 & 1028 & 8.48 \\
8 & 739 & 15.45 \\
\hline
\end{tabular}

TABLE 5: Comparison of AA desired and predicted by the BP neural network model and newff function.

\begin{tabular}{lccc}
\hline Test no. & Desired AA $(\mathrm{cm})$ & BP model AA $(\mathrm{cm})$ & Error $(\%)$ \\
\hline 1 & 2.35 & 2.78 & 18.69 \\
2 & 3.85 & 3.99 & 3.66 \\
3 & 5.28 & 5.31 & 0.70 \\
4 & 4.44 & 4.57 & 3.1 \\
5 & 2.90 & 3.25 & 12.16 \\
6 & 1.27 & 1.30 & 2.44 \\
7 & 4.18 & 4.55 & 8.95 \\
8 & 2.41 & 2.57 & 6.79 \\
9 & 5.47 & 5.94 & 8.70 \\
10 & 3.04 & 3.71 & 22.27 \\
\hline
\end{tabular}

for verifying or testing network capabilities in modeling the vehicle vibration.

Therefore, the general network structure is supposed to be $4-n-1$, which implies 4 neurons in the input layer, $n$ neurons in the hidden layer, and 1 neuron in the output layer. Then, by varying the number of hidden neurons, different network configurations are trained, and their performances are checked. The results are shown in Table 4.

For training problem, equal learning rate and momentum constant of $\eta=\alpha=0.85$ were used [16]. Also, error stopping criterion was set at $E=0.01$, which means that training epochs continued until the mean square error fell beneath this value. Both the required iteration numbers and mapping
TABLE 6: Comparison of AA desired and predicted by the BP neural network model and newcf function.

\begin{tabular}{lccc}
\hline Test no. & Desired AA $(\mathrm{cm})$ & BP model AA $(\mathrm{cm})$ & Error $(\%)$ \\
\hline 1 & 2.35 & 2.54 & 8.34 \\
2 & 3.85 & 4.36 & 13.26 \\
3 & 5.28 & 5.76 & 9.10 \\
4 & 4.44 & 4.80 & 8.15 \\
5 & 2.90 & 3.23 & 11.56 \\
6 & 1.27 & 1.29 & 1.64 \\
7 & 4.18 & 4.94 & 18.29 \\
8 & 2.41 & 2.54 & 5.65 \\
9 & 5.47 & 5.66 & 3.49 \\
10 & 3.04 & 3.38 & 11.36 \\
\hline
\end{tabular}

TABLE 7: Comparison of AA desired and predicted by the BP neural network model and newfftd function.

\begin{tabular}{lccc}
\hline Test no. & Desired AA $(\mathrm{cm})$ & BP model AA $(\mathrm{cm})$ & Error $(\%)$ \\
\hline 1 & 2.35 & 2.58 & 9.86 \\
2 & 3.85 & 4.75 & 23.39 \\
3 & 5.28 & 5.80 & 9.90 \\
4 & 4.44 & 5.09 & 14.67 \\
5 & 2.90 & 3.11 & 7.49 \\
6 & 1.27 & 1.47 & 16.24 \\
7 & 4.18 & 4.54 & 8.79 \\
8 & 2.41 & 2.45 & 2.07 \\
9 & 5.47 & 6.04 & 10.47 \\
10 & 3.04 & 3.08 & 1.39 \\
\hline
\end{tabular}

TABLE 8: Comparison of AA desired and predicted by the BP neural network model and newelm function.

\begin{tabular}{lccc}
\hline Test no. & Desired AA $(\mathrm{cm})$ & BP model AA $(\mathrm{cm})$ & Error $(\%)$ \\
\hline 1 & 2.35 & 2.48 & 5.77 \\
2 & 3.85 & 3.90 & 1.37 \\
3 & 5.28 & 5.44 & 3.14 \\
4 & 4.44 & 4.47 & 0.9 \\
5 & 2.90 & 3.21 & 10.88 \\
6 & 1.27 & 1.29 & 1.80 \\
7 & 4.18 & 4.64 & 11.15 \\
8 & 2.41 & 2.57 & 6.79 \\
9 & 5.47 & 5.69 & 4.17 \\
10 & 3.04 & 3.15 & 3.62 \\
\hline
\end{tabular}

performances were examined for these networks. As the error criterion for all networks was the same, their performances are comparable. As a result, from Table 4, the best network structure of BP model is picked to have 6 neurons in the hidden layer with the average verification errors of $4.95 \%$ in amplitude acceleration over the 10 numerical verification data sets. Tables 5, 6, 7 and 8 show the comparison of desired and predicted values for amplitude acceleration in verification cases with different functions.

Figure 4 illustrates the convergence of the output error (mean square error) with the number of iterations (epochs) 
TABLE 9: The $\left(R^{2}\right)$ values for AA with various neurons in the hidden layer.

\begin{tabular}{lccccccrrr}
\hline $\begin{array}{l}\text { Number of } \\
\text { hidden neurons }\end{array}$ & LM & BFG & RP & SCG & CGB & CGF & CGP & OSS & GDX \\
\hline 4 & 0.9977 & 0.9888 & 0.9819 & 0.9957 & 0.9738 & 0.9918 & 0.9905 & 0.9913 & 0.9891 \\
5 & 0.9981 & 0.9676 & 0.992 & 0.9909 & 0.9942 & 0.9864 & 0.9846 & 0.9969 & 0.9916 \\
6 & 0.9999 & 0.9981 & 0.9942 & 0.9578 & 0.9986 & 0.9978 & 0.9681 & 0.9963 & 0.9945 \\
7 & 0.9985 & 0.9998 & 0.9967 & 0.9996 & 0.9996 & 0.9995 & 0.9595 & 0.9890 & 0.9856 \\
8 & 0.9967 & 0.9928 & 0.9994 & 0.9998 & 0.9998 & 0.9698 & 0.9898 & 0.9898 & 0.9881 \\
\hline
\end{tabular}

TABLE 10: The results of the variable training methods in the BPN with newelm function.

\begin{tabular}{lcccc}
\hline Acronym & $\begin{array}{c}\text { Epoch in } \\
\text { goal }\end{array}$ & Error goal & Train time (s) & Test time (s) \\
\hline LM & 1753 & Met & 28.7240 & 0.054526 \\
BFG & 2528 & Met & 77.4824 & 0.046583 \\
RP & 3000 & Not met & 67.6773 & 0.046466 \\
SCG & 3000 & Not met & 101.3281 & 0.044047 \\
CGB & 2932 & Not met & 118.1020 & 0.052230 \\
CGF & 2125 & Not met & 88.8947 & 0.046125 \\
CGP & 2302 & Not met & 94.6653 & 0.046852 \\
OSS & 3000 & Not met & 112.0710 & 0.046677 \\
GDX & 3000 & Not met & 61.2435 & 0.046046 \\
\hline
\end{tabular}

TABLE 11: The results of the variable training methods in the BPN with newcf function.

\begin{tabular}{lcccc}
\hline Acronym & $\begin{array}{c}\text { Epoch in } \\
\text { goal }\end{array}$ & Error goal & Train time (s) & Test time (s) \\
\hline LM & 1251 & Met & 38.7261 & 0.057241 \\
BFG & 2018 & Met & 97.4814 & 0.042678 \\
RP & 2439 & Not met & 49.1627 & 0.048146 \\
SCG & 1980 & Not met & 33.1907 & 0.042047 \\
CGB & 3232 & Not met & 128.1590 & 0.055130 \\
CGF & 3025 & Not met & 108.1528 & 0.056985 \\
CGP & 2100 & Not met & 67.1503 & 0.044152 \\
OSS & 1000 & Met & 59.0112 & 0.058677 \\
GDX & 3230 & Not met & 67.1205 & 0.056046 \\
\hline
\end{tabular}

during training of the chosen 4-6-1 BP network. After 1753 epochs, the MSE between the desired and predicted outputs becomes less than 0.01 . At the beginning of the training, the output from the network is far from the target value. However, the output slowly and gradually converges to the target value with more epochs and the network learns the input/output relation of the training samples. The regression value $\left(R^{2}\right)$ of the output variable values for the test data set for various neurons in hidden layer is shown in Table 9. It should be noted that these data were completely unknown to the network. The closer this value is to unity, the better is the prediction accuracy. The best $\left(R^{2}\right)$ value obtained is 0.9999 , and it is obtained from the LM algorithm by 6 neurons in hidden layer.
TABLE 12: The results of the variable training methods in the BPN with newff function.

\begin{tabular}{lcccc}
\hline Acronym & $\begin{array}{c}\text { Epoch in } \\
\text { goal }\end{array}$ & Error goal & Train time (s) & Test time (s) \\
\hline LM & 1293 & Met & 40.1450 & 0.054789 \\
BFG & 1528 & Met & 88.1024 & 0.056673 \\
RP & 1210 & Met & 100.6773 & 0.056906 \\
SCG & 4000 & Not met & 97.9497 & 0.065237 \\
CGB & 3745 & Not met & 138.7890 & 0.062230 \\
CGF & 3450 & Not met & 112.8964 & 0.053565 \\
CGP & 3162 & Not met & 100.1333 & 0.056907 \\
OSS & 3400 & Not met & 160.1489 & 0.066677 \\
GDX & 2000 & Not met & 43.4465 & 0.056044 \\
\hline
\end{tabular}

TABLE 13: The results of the variable training methods in the BPN with newfftd function.

\begin{tabular}{lcccc}
\hline Acronym & $\begin{array}{c}\text { Epoch in } \\
\text { goal }\end{array}$ & Error goal & Train time (s) & Test time (s) \\
\hline LM & 2167 & Met & 43.1808 & 0.061301 \\
BFG & 19018 & Met & 64.1920 & 0.058573 \\
RP & 1890 & Met & 99.7452 & 0.064916 \\
SCG & 3786 & Not met & 39.1877 & 0.061937 \\
CGB & 4150 & Not met & 134.3904 & 0.067220 \\
CGF & 1129 & Not met & 102.5594 & 0.057345 \\
CGP & 2001 & Not met & 100.1333 & 0.055897 \\
OSS & 4744 & Not met & 80.9358 & 0.060127 \\
GDX & 3190 & Not met & 38.2065 & 0.059391 \\
\hline
\end{tabular}

In Tables 10,11, 12, and 13, the results of training the network using nine different training algorithms by 6 neurons in the hidden layer and logsig-purlin activation function are summarized. Each entry in the table represents 10 different trials, where different random initial weights are used in each trial.

\section{Conclusions and Summary}

In this paper, four supervised neural networks have been used for the vehicle vibrations. Based on the test results of each network with some data set, different from those used in the training phase, it was shown that newelm function neural model has superior performance than newff, newcf, 


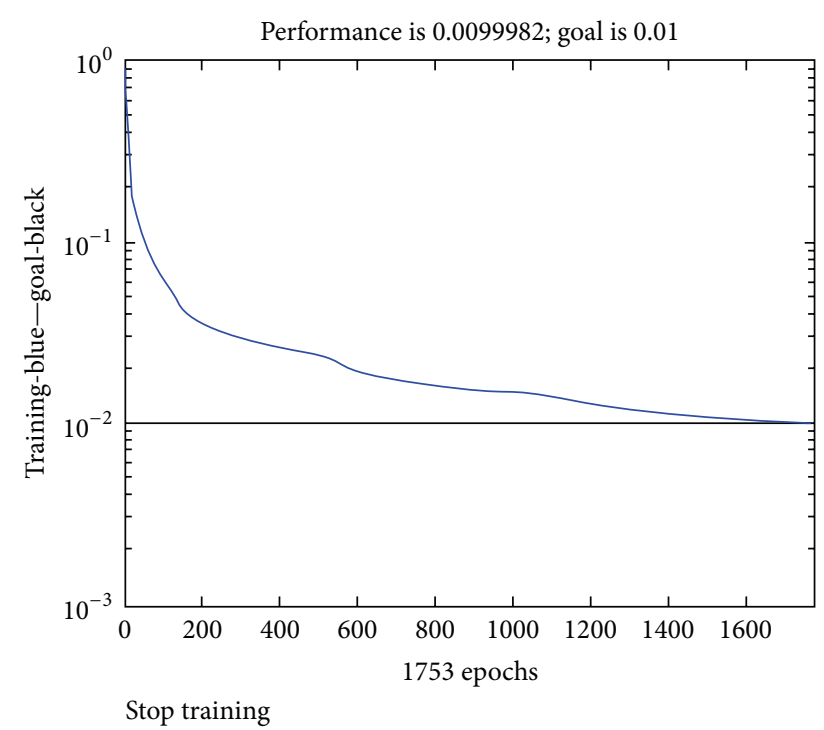

FIGURE 4: Learning behavior of the BP neural network model.

and newfftd functions and can predict outputs in a wide range of vehicle vibration conditions with reasonable accuracy.

In sum, the following items can also be mentioned as the general findings of the present research.

(1) The BP neural networks are capable of constructing models using only desired data, describing proper vehicle vibration behavior.

(2) BP neural network with newelm function which possesses the privileges of rapid learning, easy convergence, and less error with respect to other functions has better generalization power and is more accurate for this particular case. This selection was done according to the results obtained in the verification phase.

(3) Velocity is the dominant factor among other input parameters, so that increasing velocity in a constant level of damping ratio and natural frequency increases the acceleration amplitude.

\section{References}

[1] R. Guclu and K. Gulez, "Neural network control of seat vibrations of a non-linear full vehicle model using PMSM," Mathematical and Computer Modelling, vol. 47, no. 11-12, pp. 1356-1371, 2008.

[2] S. Li, S. Yang, and W. Guo, "Investigation on chaotic motion in hysteretic non-linear suspension system with multi-frequency excitations," Mechanics Research Communications, vol. 31, no. 2, pp. 229-236, 2004.

[3] Q. Zhu and M. Ishitobi, "Chaotic vibration of a nonlinear fullvehicle model," International Journal of Solids and Structures, vol. 43, no. 3-4, pp. 747-759, 2006.

[4] G. Litak, M. Borowiec, M. I. Friswell, and K. Szabelski, "Chaotic vibration of a quarter-car model excited by the road surface profile," Communications in Nonlinear Science and Numerical Simulation, vol. 13, no. 7, pp. 1373-1383, 2008.
[5] T. Ishihama, H. Masao, and M. Seto, "Vehicle vibration reduction by transfer function phase control on hydraulic engine mounts," JSME International Journal C, vol. 37, no. 3, pp. 536$541,1994$.

[6] T. Y. Yi and P. E. Nikravesh, "A method of identify vibration characteristics of modified structures for flexible vehicle dynamics," Proceedings of the Institution of Mechanical Engineers D, vol. 216, no. 1, pp. 55-63, 2002.

[7] B. Liang, D. Zhu, and Y. Cai, "Dynamic analysis of the vehiclesubgrade model of a vertical coupled system," Journal of Sound and Vibration, vol. 245, no. 1, pp. 79-92, 2001.

[8] D. A. Linkens and H. O. Nyongesa, "Learning systems in intelligent control: an appraisal of fuzzy, neural and genetic algorithm control applications," IEE-Proceedings of the Control Theory Applications, vol. 143, no. 4, pp. 367-386, 1996.

[9] V. Rouillard and M. A. Sek, "Simulation of non-stationary vehicle vibrations," Proceedings of the Institution of Mechanical Engineers D, vol. 215, no. 10, pp. 1069-1075, 2001.

[10] Ş. Yildirim and I. Uzmay, "Neural network applications to vehicle's vibration analysis," Mechanism and Machine Theory, vol. 38, no. 1, pp. 27-41, 2003.

[11] Ş. Yildirim and I. Uzmay, "Statistical analysis of vehicles' vibration due to road roughness using Radial Basis artificial Neural Network," Applied Artificial Intelligence, vol. 15, no. 4, pp. 419-427, 2001.

[12] W. Q. Zhu, Random Vibration, Academic Press, Beijing, China, 1992.

[13] D. E. Newland, An Introduction to Random Vibration, Spectral and Wavelet Analysis, Longman Scientific and Technical Group, England, UK, 3rd edition, 1993.

[14] I. Uzmay, "Investigation of vehicle vibrations due to random excitation by road roughness," in Proceedings of Second National Machine Design and Production Conference, pp. 159-165, 1986.

[15] H. Zhang, W. Wu, and M. Yao, "Boundedness and convergence of batch back-propagation algorithm with penalty for feedforward neural networks," Neurocomputing, vol. 89, pp. 141-146, 2012.

[16] H. Shao and G. Zheng, "Convergence analysis of a backpropagation algorithm with adaptive momentum," Neurocomputing, vol. 74, no. 5, pp. 749-752, 2011.

[17] D. Gao, Y. Kinouchi, K. Ito, and X. Zhao, "Neural networks for event extraction from time series: a back propagation algorithm approach," Future Generation Computer Systems, vol. 21, no. 7, pp. 1096-1105, 2005.

[18] H. Demuth and M. Beale, Matlab Neural Networks Toolbox, User's Guide, Copyright 1992-2001, The Math Works, Inc, http://www.mathworks.com .

[19] D. Ballabio and M. Vasighi, "A Matlab toolbox for self organizing maps and supervised neural network learning strategies," Chemometrics and Intelligent Laboratory Systems, vol. 118, pp. 24-32, 2012. 

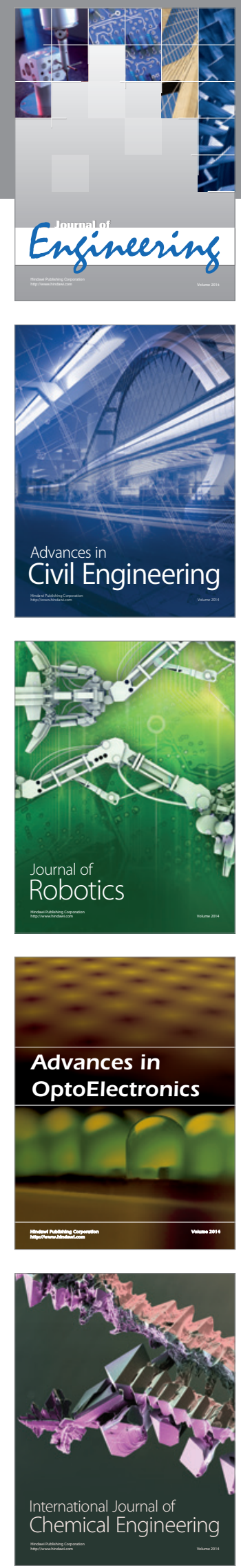

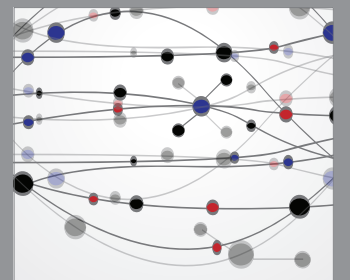

The Scientific World Journal
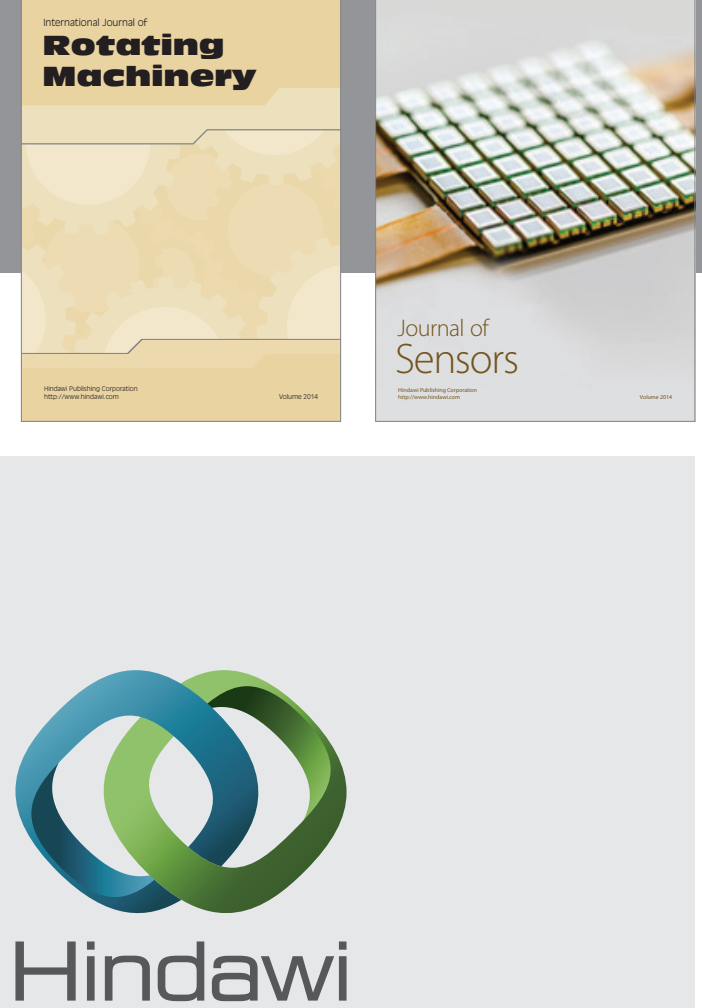

Submit your manuscripts at http://www.hindawi.com
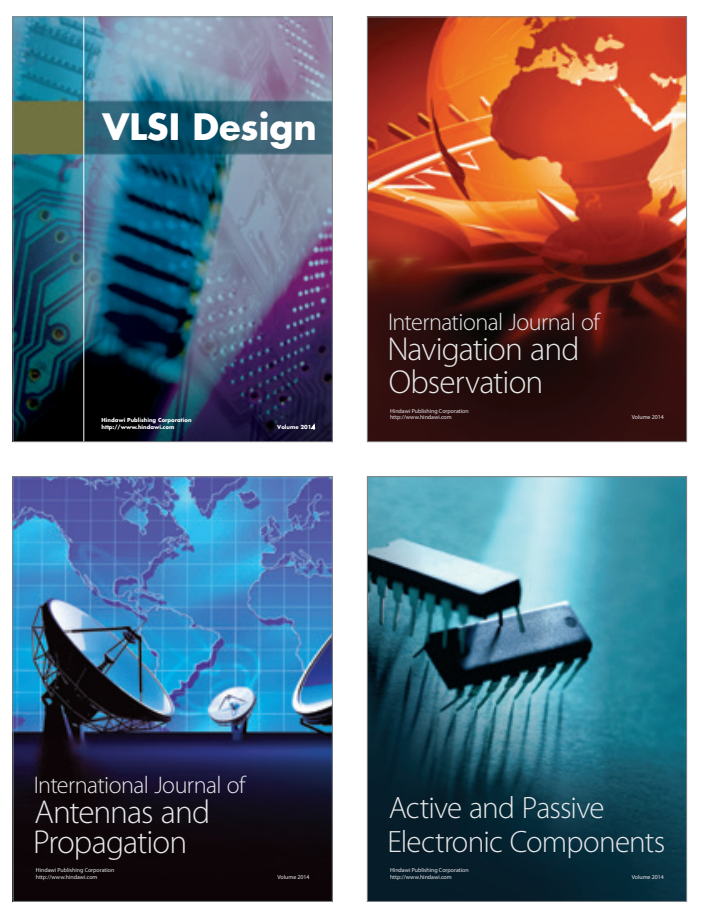
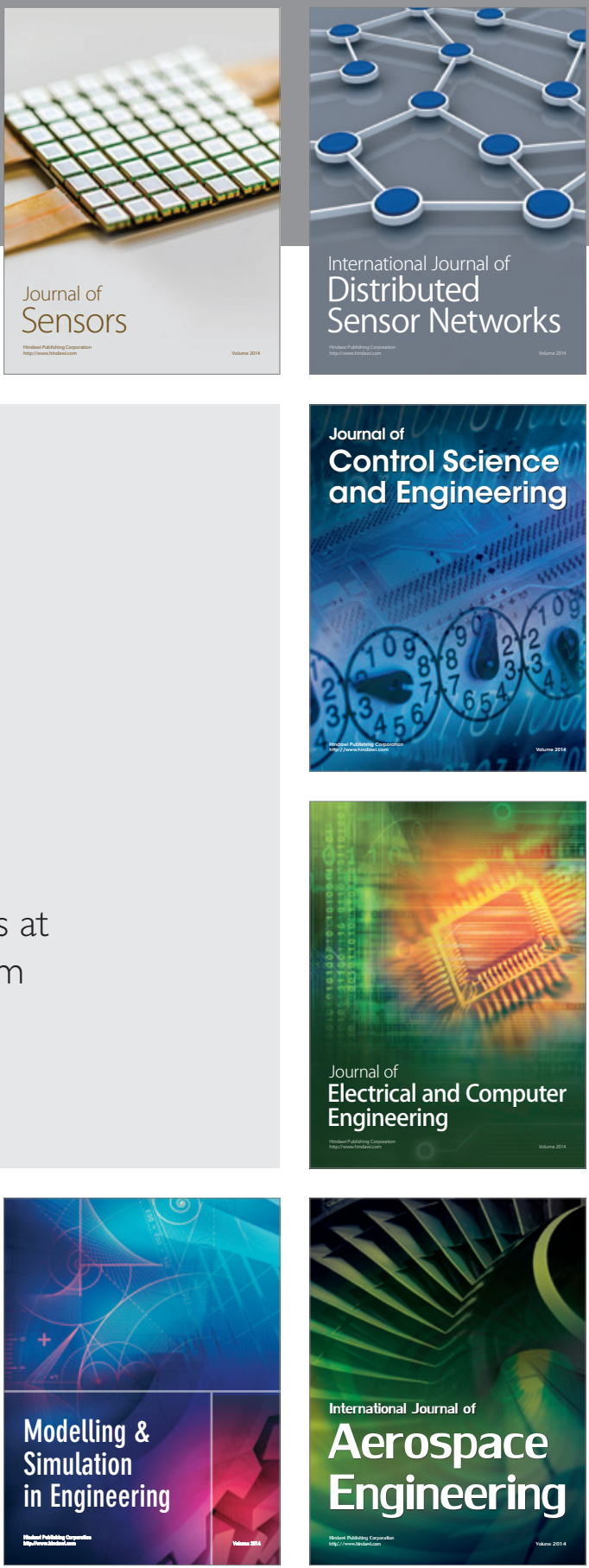

Journal of

Control Science

and Engineering
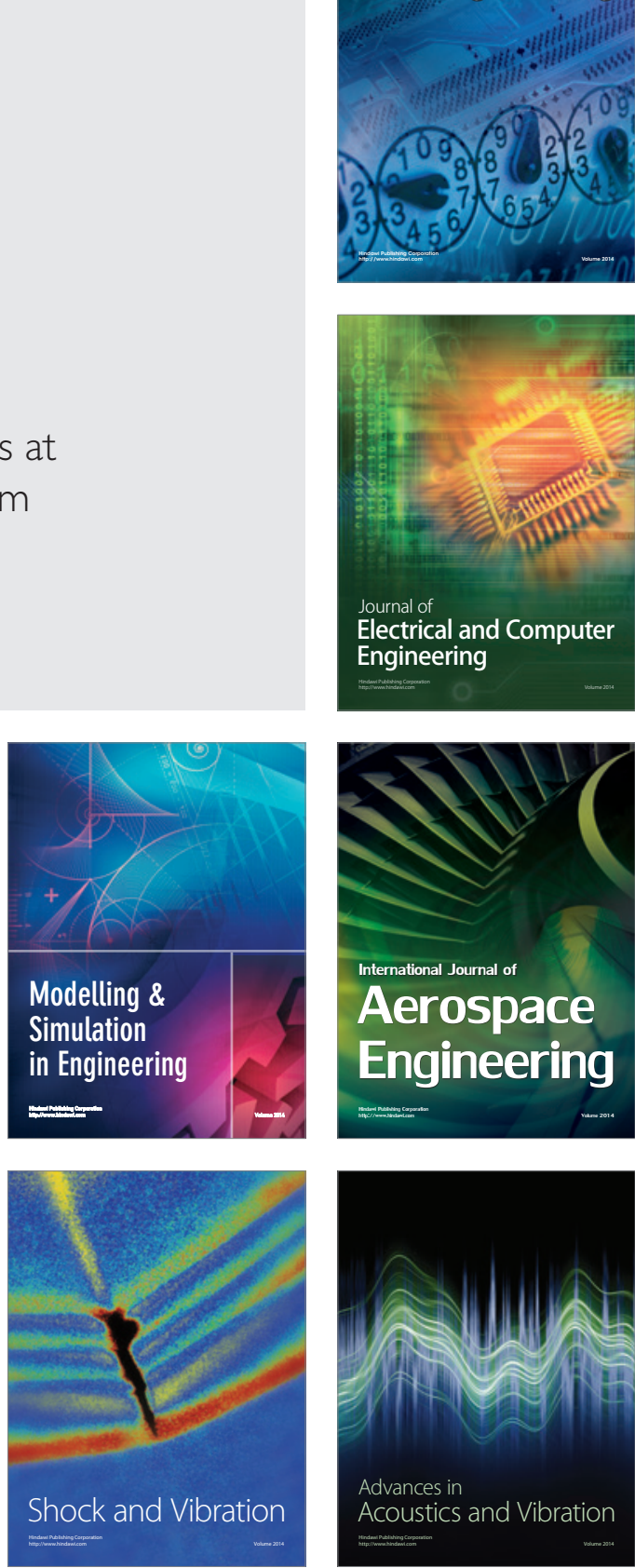